\title{
Clinical Profile and Outcome of Dengue Fever in Tertiary Care Hospital at Kerala
}

\author{
P. Leela Kumari ${ }^{\odot}$ \\ Associate Professor, Department of General Medicine, Mount Zion Medical College Hospital Ezhamkulam, Adoor, Pathanamthitta, Kerala, India.
}

\section{Abstract}

Background: Among all flaviviruses, Dengue is the most common fever. Every year 2-5 lakh cases of Dengue hemorrhagic fever occur. The objective of the study was to evaluate the clinical profile of patients with Dengue fever at a hospital in Kerala. Subjects and Methods: Patients with more than 12 years of age and those admitted with fever, headache, and myalgia were assessed with other clinical characteristics (warning signs of dengue fever, signs of hemorrhage, signs of plasma leakage and signs of shock). Laboratory examinations such as Hb, total count, differential count, platelet count, packed cell volume, peripheral smear for the malarial parasite, and liver function test (including S. protein) and renal function test were carried out. Blood coagulation profiles such as Bleeding Time, Clotting Time, Prothrombin Time and aPTT were performed. Radiological findings like X-ray chest and USG abdomen were also performed. Results: Consequently dengue fever is ordinary in young adults. In the current study, males were more pretentious than females. Males were affected by $63.7 \%$ while females are affected by $36.3 \%$. The most usual indication was fever (94.8\%) experienced in all patients followed by headache (41.3\%), Generalized weakness (77.5\%), myalgia (51.7\%), vomiting (57.7\%). Predominant organ involvement was hepatic (transaminases, jaundice) followed by gastrointestinal (abdominal pain, distension, loose stools, vomiting, ascites) systems. The total survivors are 86(74.1\%) whereas 30(25.8\%) were non-survivors. Conclusion: Dengue fever usually affects male and young populace. Headache, fever, myalgia are ordinary at hand grumble however in the recent few years, the world has observed the diverse experimental appearance of the dengue fever in dissimilar epidemics, even in the same regions and even with the period of times.

Keywords: Dengue Fever, Flaviviruses, Hemorrhagic Fever

Corresponding Author: P. Leela Kumari, Associate Professor, Department of General Medicine, Mount Zion Medical College Hospital Ezhamkulam, Adoor, Pathanamthitta, Kerala, India.

E-mail: leelakumari9@yahoo.com

Received: 30 September 2020

Revised: 17 November 2020

Accepted: 26 November 2020

Published: 26 December 2020

\section{Introduction}

Dengue Fever (DF) and Dengue Hemorrhagic Fever (DHF) have come out as a worldwide public health issue. Infect dengue is hyperendemic in many urban, peri-urban and rural areas with frequent epidemics. ${ }^{[1]}$

Dengue fever is caused by an arthropod-borne virus of genus flavivirus belonging to the family Flaviviridae, presenting with a wide clinical spectrum that may range from asymptomatic fever to dreaded difficulty such as hemorrhagic fever and shock. ${ }^{[2]}$

The epidemiology of dengue relies on a complex association between epidemiologic factors viz. host, agent and environment. ${ }^{[3]}$ The agent, dengue virus is classified under flavivirus. There are four genetically associated however antigenically discrete DEN serotypes (DENV-1, DENV-2, DENV-3 and DENV-4) all of which are widespread in India.
Dengue viruses are conveyed from a contaminated person to others. Aeaegypti breeds almost entirely in domestic manmade water vessels are found in and around households. The populace of mosquitoes varies with rainfall and water storage. Its existence is affected by temperature and clamminess. The virus contaminates humans and lower primates also. The mosquito becomes contaminated when it takes a blood meal from the person during the febrile phase of sickness. Post extrinsic incubation period of 8 to 10 days, mosquito grows to be infected and then it bites another person and inject saliva into through which virus enters, 4-7 days of inherent incubation period dengue happens unexpectedly.

The occurrence of dengue is mounting in India from 6.34 per million inhabitants between 1998 and 2009 to per million populace between 2010 and 2014. ${ }^{[4]}$ Appearance of dengue fever can differ from malaise, fatigability as a part viral prodrome to shock and multi-organ dysfunction 
syndrome as a part of severe illness. ${ }^{[5]}$ Results of patients with dengue fever admitted to critical care units have been calculated less often. Prognostic factors which settle on the medical results of gravely ill patients with dengue fever linger indistinguishable. ${ }^{[6]}$ This viral infection has a broad quantifiable gamut varying from asymptomatic ailment to undifferentiated fever (or viral syndromes), classical dengue fever (DF), dengue hemorrhagic fever (DHF), or dengue shock syndrome (DSS) and prolonged dengue syndrome (EDS) ${ }^{[7]}$ According to the World Health Organization (WHO) criteria 2009, patients are categorized as stern dengue if they have a demonstration of relentless plasma leakage, severe hemorrhage, or severe organ mutilation. Dengue may be identified by microbiological laboratory testing for viral antigen detection or specific antibodies (serology), nucleic acid detection by PCR. ${ }^{[8]}$ Timely recognition depends on the clinical notion and corroboratory laboratory facts can help to restrict a number of cases that advance to extend snag with timely interference. Thus this study was carried out to classify and study the clinical profile of the sufferers of dengue.

\section{Subjects and Methods}

The study was performed on 100 cases of dengue fever in the Department of medicine from august-2018 to May 2019. The case definition was based on consent, compatible clinical history and examination based on WHO criterion, established by positive serology to dengue fever. Patients with more than 12 years of age and those admitted with fever, headache, and myalgia were assessed with other clinical characteristics (warning signs of dengue fever, signs of hemorrhage, signs of plasma leakage and signs of shock). Laboratory examinations such as $\mathrm{Hb}$, total count, differential count, platelet count, packed cell volume, peripheral smear for the malarial parasite, and liver function test (including S. protein) and renal function test were carried out. Blood coagulation profiles such as Bleeding Time, Clotting Time, Prothrombin Time and aPTT were performed. Radiological findings like X-ray chest and USG abdomen were also performed. Other examinations like S. widal, blood for culture were performed too. Brain imaging, fundus examination were carried out as and when required. Serology examinations for dengue fever (NS1, IgM, and $\mathrm{IgG}$ ) were also carried out. Dengue fever with warning signs and severe dengue between 1st July and 1st December 2015 NS1-Antigen Strip ${ }^{\circledR}$ ELISA (BioRad), IgM and IgGCapture ELISA Panbio ${ }^{\circledR}$ (Alere $^{\mathrm{TM}}$ ) were used for dengue serology testing. Patient management was as per the guidelines laid by WHO. Surviving Sepsis Campaign guidelines and established standards were applied in intensive care practice. Demographics counting age and gender division, signs and symptoms, SOFA and APACHE II scores were stored. Upshot details together with organ association and endurance to hospital discharge were counted. Statistical Analysis was performed by SPSS version 13.0 used for data analysis.

\section{Results}

A total number of 116 patients with dengue fever were registered in the study. In the current study, most widespread age group was between 20-29 years with the mean age being 26.5 years. Consequently, dengue fever is ordinary in young adults. In the current study, males were more pretentious than females. Males were affected by $63.7 \%$ while females are affected by $36.3 \%$. [Figure 1] The most usual indication was fever (94.8\%) experienced in all patients followed by headache (41.3\%), Generalized weakness (77.5\%), myalgia (51.7\%), vomiting (57.7\%) [Figure $2 \& 3$ ]. Predominant organ involvement was hepatic (transaminases, jaundice) followed by gastrointestinal (abdominal pain, distension, loose stools, vomiting, ascites) systems. The total survivors are $86(74.1 \%)$ whereas $30(25.8 \%)$ were non-survivors. Hematological participation was predominantly thrombocytopenia and raised hematocrit. Respiratory system involvement had ARDS and Pleural effusion. CNS manifestations incorporated intracranial bleed, cerebral infarction and cerebellitis (in one patient). Factors connected with transience were exposed in table 1 and patients with abnormal laboratory ruling in dengue fever were displayed in [Table no. 2].

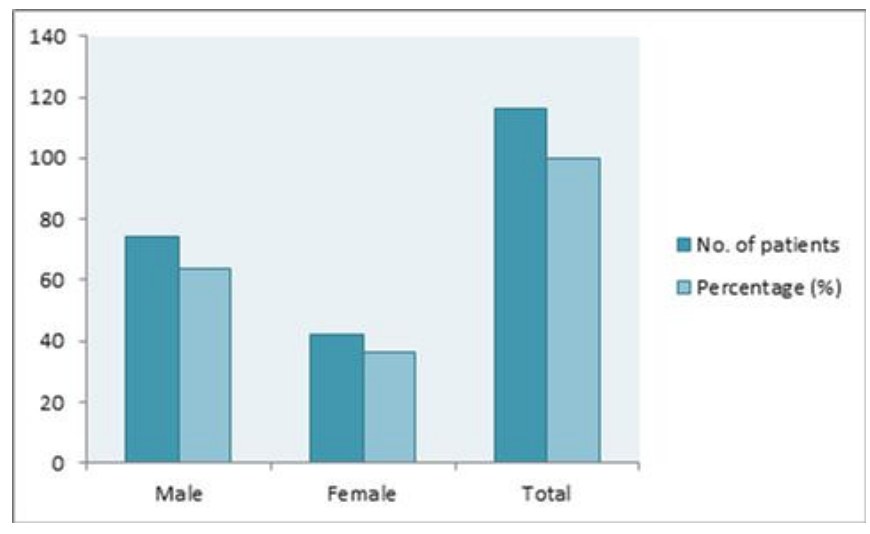

Figure 1: Sex distribution of patients $(\mathrm{N}=116)$.

\section{Discussion}

We experienced in the study that dengue fever patients present with varying manifestations. Fever was the common clinical feature with which patients present to the hospital followed by headache $(41.3 \%)$, Generalized weakness $(77.5 \%)$, myalgia (51.7\%), vomiting (57.7\%). In Kerala study and in Northeast India study also fever was a most common indication were experienced in $96.8 \%$ and $98.1 \%$ cases correspondingly. ${ }^{[9,10]}$ 


\begin{tabular}{|c|c|c|c|c|}
\hline Variable & Survivors $(\mathrm{n}=86)$ & $\begin{array}{l}\text { Non survivors }(\mathrm{n}= \\
\text { 30) }\end{array}$ & OR (IQR) & p-value \\
\hline Jaundice & $9(10.4 \%)$ & $12(40 \%)$ & $5.69(1.74-19.0)$ & 0.002 \\
\hline $\begin{array}{l}\text { ARDS(PaO2/FiO2 } \\
<200)\end{array}$ & $24(27.9 \%)$ & $25(83.3 \%)$ & $12.3(3.2-53.1)$ & $<0.01$ \\
\hline $\begin{array}{l}\text { Acute kidney injury } \\
\text { (AKI) }\end{array}$ & $17(19.7 \%)$ & $29(96.6 \%)$ & 78.31 (9.51-629) & $<0.01$ \\
\hline Shock & $18(20.9 \%)$ & $28(93.3 \%)$ & $72.9(9.80-572)$ & $<0.01$ \\
\hline $\begin{array}{l}\text { Central nervous sys- } \\
\text { tem }\end{array}$ & $22(25.5 \%)$ & $12(40 \%)$ & $1.81(0.61-6.21)$ & 0.238 \\
\hline Respiratory & $55(63.9 \%)$ & $28(93.3 \%)$ & $12.02(2.9-87.9)$ & $<0.05$ \\
\hline Gastrointestinal & $51(59.3 \%)$ & $28(93.3 \%)$ & $13.9(1.69-104)$ & $<0.05$ \\
\hline
\end{tabular}

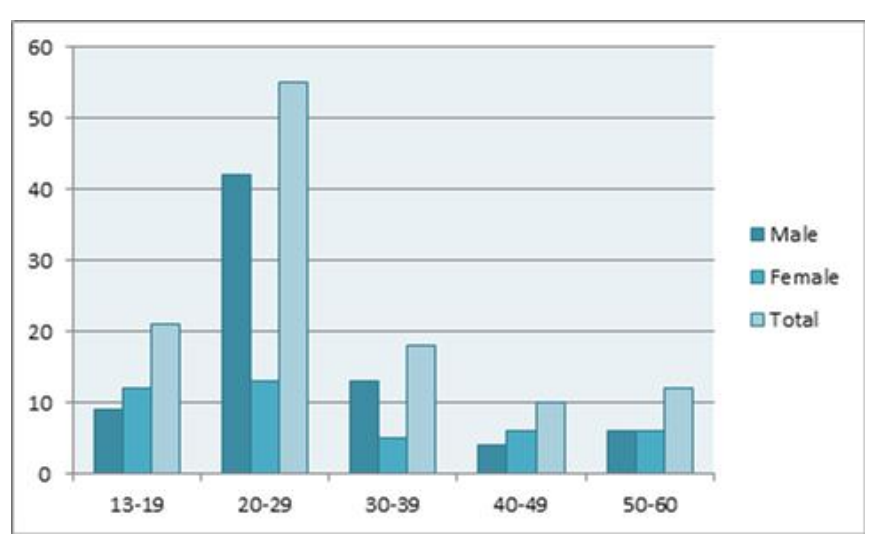

Figure 2: Age distribution of patients ( $\mathrm{N}=116)$.

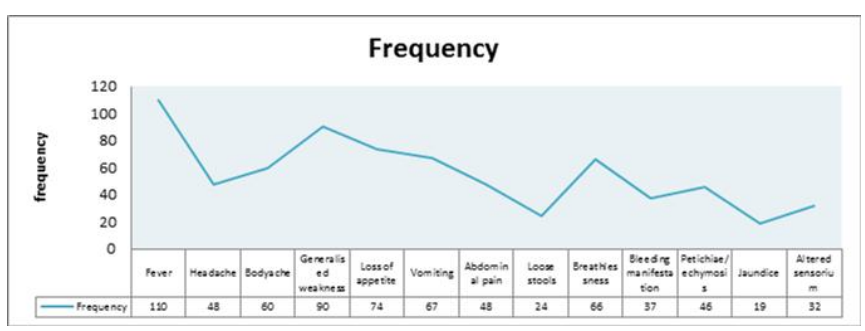

\section{Figure 3: F requency of patients with Clinical features}

In the study of Itoda $\mathrm{I}$ et al, in Japan and Northeast India study headache experienced in $90 \%$ and $93.4 \%$ cases correspondingly. ${ }^{[10,11]}$ In the Northeast India study myalgia was observed in $81.3 \%$ of cases. ${ }^{[10]}$

The duration of stay of hospitalization was more in acute dengue cases equivalent to many types of research. Improvement after 1week of ill health in the preponderance of cases (64\%), which somewhat diverse from other studies where the

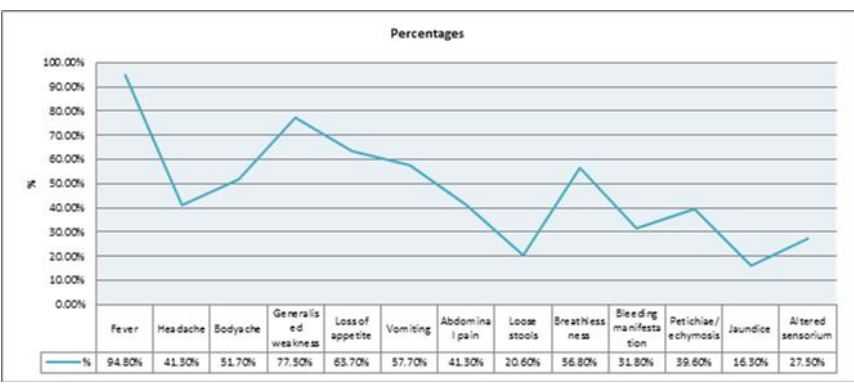

Figure 4: Percentages of patients with Clinical features

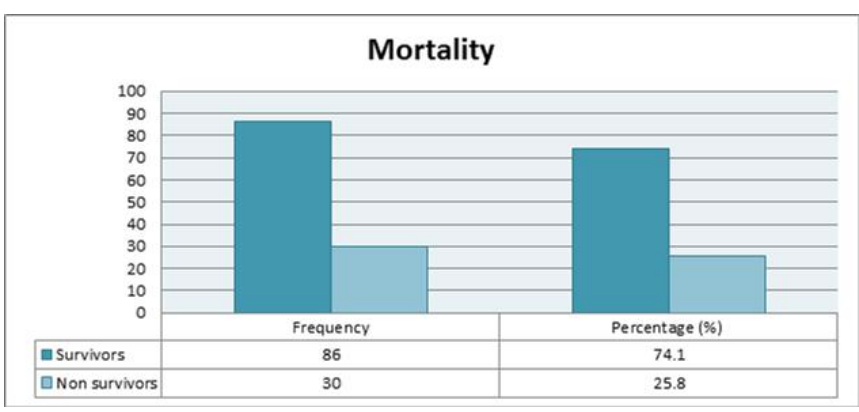

Figure 5: Frequency and percentages of survivors and non-survivors

mean extent of stay 5-6 days. ${ }^{[12,13]}$ According to our study most of them obtained with thrombocytopenia $(79.3 \%)$ at admittance than leucopenia, comparable to some studies ${ }^{[14]}$ and NS1 antigen positive cases $(61.5 \%)$ found more than IgM positive. ${ }^{[15]}$ Multiorgan participation in dengue is very well-acknowledged. In our study, we found Hepatic participation among $89.5 \%$. AST and ALT were appreciably high among nonsurvivors. Likewise, in a study by Nayak fetal liver association was noted among 97.33\%, AST and ALT 


\begin{tabular}{lll|}
\hline \multicolumn{2}{|l|}{ Table 2: Abnormal laboratory findings in dengue fever patients } \\
\hline $\begin{array}{l}\text { Laboratory } \\
\text { findings }\end{array}$ & No. of patients & Percentage (\%) \\
\hline $\mathrm{Hb}(\leq 10 \mathrm{gm} \%)$ & 8 & 6.8 \\
$\begin{array}{l}\text { Leucopenia } \\
(<4000 / \mathrm{ul})\end{array}$ & 65 & 56.0 \\
$\begin{array}{l}\text { Thrombocytopenia } \\
(<1.0 \text { lac/ul })\end{array}$ & 92 & 79.3 \\
$\begin{array}{l}\text { Haematocrit } \\
(\geq 45 \%)\end{array}$ & 17 & 14.6 \\
\hline PT $(>14 \mathrm{sec})$ & 5 & 4.3 \\
\hline aPTT $(>40$ sec $)$ & 5 & 4.3 \\
\hline INR $(>1.2)$ & 5 & 4.3 \\
\hline $\begin{array}{l}\text { S.creatinine } \\
(>1.2 \text { mg\%) }\end{array}$ & 10 & 8.6 \\
\hline
\end{tabular}

were appreciably high among dengue hemorrhagic fever and dengue shock syndrome group. ${ }^{[16]}$ Respiratory and gastrointestinal involvements had augmented the jeopardy of mortality. AKI and moderate-severe ARDS (withPFratio<200) were considerably high among nonsurvivors. Likewise, Shock and Lactates were also noted to be considerably elevated among non-survivors. The study by Thana chart wet et al displayed considerably elevated lactates among sufferers with organ failure and shock. ${ }^{[17]}$ In a study by Chen et al moderate ARDS (PF Ratio <200) followed by shock $(\mathrm{SBP}<90 \mathrm{mmHgorMAP}<65)$ and multi-organ failure were significant among non-survivors. ${ }^{[18]}$ A study by Jain et al. portrayed liver and renal connection, ARDS, Shock necessitating vasopressor, metabolic acidosis as predictors of mortality. ${ }^{[19]}$ A study done in Cuttack, India had shown predominant organ failure was coagulation (48\%). Respiratory failure was $19 \%$ and cardiovascular failure was $8 \% .{ }^{[20]}$ Mortality has been displayed to be high among the age group of $>60$ years in prior studies. ${ }^{[18]} \mathrm{O}$ ur study had younger patients between 21 years and 40 years requiring ICU and mortality was noted to be high among females between the age group of 21-40 years. In an exposition cohort study from Brazil mortality of severe dengue was noted to be $3.1 \%$ over a period of 14 years. Age $>55$ years was associated with increased mortality. ${ }^{[21]}$ In a Chinese study, female sex and age group of 30-39 years was associated with increased risk of dengue hemorrhagic fever and mortality especially with dengue 2 serotype. ${ }^{[22]}$ Pangetal noted that adult patients fitting into 50 to 59year age group of patients with diabetes had 5 times amplified hazard for ICU admission and enlarged mortality as evaluated to patients who are younger than 30 years and without diabetes. ${ }^{[23]}$ Another study was performed in Singapore between 2007 and 2008 during the dengue sero-type 2 epidemic observed that female sex, age group 30-49 years and presence of diabetes or hypertension were linked with an augmented danger of dengue hemorrhagic fever. ${ }^{[24]}$

\section{Conclusion}

Dengue fever usually has an effect on male and young populace. Headache, fever, myalgia are ordinary at hand grumble however in the recent few years, the world has observed the diverse experimental appearance of the dengue fever in dissimilar epidemics, even in the same regions and even with the period of times. Where some recognized characteristics are still obvious, few atypical characteristics are noted from quite a lot of parts of the world. Lactatemia, transaminases, elevated ailment sternness scores (APACHE II and SOFA), Shock, AKI and $\mathrm{PaO} 2 / \mathrm{FiO} 2$ ratio less than 200 were connected with augmented mortality.

\section{References}

1. Shah I, Deshpande GC, Tardeja PN. The outbreak of dengue in Mumbai and predictive markers for dengue shock syndrome. J Trop Pediatr. 2004;50(5):301-306. Available from: https: //doi.org/10.1093/tropej/50.5.301.

2. Special programme for research, training in tropical diseases, and World Health Organization, dengue: guidelines for diagnosis, treatment, prevention and control, World Health Organization; 2009.

3. $; 2015$.

4. Laul A, Laul P, Merugumala V, Pathak R, Miglani U, Saxena P. Clinical Profiles of Dengue Infection during an Outbreak in Northern India. J Trop Med. 2016;2016:1-7. Available from: https://dx.doi.org/10.1155/2016/5917934.

5. Mutheneni SR, Morse AP, Caminade C, Upadhyayula SM. Dengue burden in India: recent trends and importance of climatic parameters. Emerg Microbes Infect. 2017;6(1):1-10. Available from: https://dx.doi.org/10.1038/emi.2017.57.

6. Chen CM, Chan KS, Yu WL, Cheng KC, Chao HC, Yehcy. The outcomes of patients with severe dengue admitted to intensive care units. Medicines. 2016;95(31):1-5. Available from: https: //doi.org/10.1097/md.0000000000004376.

7. Clinical Manifestations and Management of Dengue/DHF/DSS. Trop Med Health. 2011;39((4 Suppl)):837. Available from: http://dx.doi.org/10.2149/tmh.2011-S10.

8. Guzman MG, Halstaed SB, Artsob H. Dengue: a continuing global threat. Nat Rev Microbiol. 2010;8(12):7-16. Available from: https://doi.org/10.1038/nrmicro2460.

9. Daniel R, Philip R, Z A. A study of clinical profile of dengue fever in Kollam. Dengue Bulletin. 2005;29:197-202.

10. Khan SA, Dutta P, Topno R, Soni M, Mahanta J. Dengue Outbreak in a Hilly State of Arunachal Pradesh in Northeast India. Sci World J. 2014;2014:1-6. Available from: https: //dx.doi.org/10.1155/2014/584093.

11. Itoda I, Suganuma A, Totsuka K, Takasaki T, Imamura A, Negishi M, et al. Clinical Features of 62 Imported Cases of Dengue Fever in Japan. Am J Trop Med Hyg. 2006;75(3):470474. Available from: https://dx.doi.org/10.4269/ajtmh.2006. 75.470 . 
12. Mittal H, Faridi MMA, Arora SK, Patil R. Clinicohematological Profile and Platelet Trends in Children with Dengue During 2010 Epidemic in North India. The Indian Journal of Pediatrics. 2012;79(4):467-471. Available from: https://dx.doi.org/10. 1007/s12098-011-0586-7. doi:10.1007/s12098-011-0586-7.

13. Chacko B, Subramanian G. Clinical, Laboratory and Radiological Parameters in Children with Dengue Fever and Predictive Factors for Dengue Shock Syndrome. J Trop Pediatr . 2007;54(2):137-140. Available from: https://dx.doi.org/10. 1093/tropej/fmm084.

14. Joshi R, Baid V. Profile of dengue patients admitted to a tertiary care hospital in Mumbai. Turkish J Pediatr. 2011;53(6):626657.

15. Ashis SK, Shibendu G. Clinico-pathological profile in the infants and children in dengue 2012 epidemic, Kolkata. Int J Med Sci Public Health . 2014;3(1):59-59. Available from: https://dx.doi.org/10.5958/j.2319-5886.3.1.012.

16. Nayakj B, Swain SK, Panda SR. A study of multiorgandysfunction in patients with dengue and its clinicohematological correlationwithseverity. Asian J Pharm Clin Res. 2017;10(2):218 221. Available from: https://doi.org/10.22159/ajpcr.2017. v10i2.15332.

17. Thanachartwet V, Desakorn V, Sahassananda D, Jittmittraphap A, Oer-areemitr N, Osothsomboon S, et al. Serum Procalcitonin and Peripheral Venous Lactate for Predicting Dengue Shock and/or Organ Failure: A Prospective Observational Study. PLOS Negl Trop Dis. 2016;10(8):e0004961-e0004961. Available from: https://dx.doi.org/10.1371/journal.pntd.0004961.

18. Chen CM, Chan KS, Yu WL, Cheng KC, Chao HC, Yehcy. The outcomes of patients with severe dengue admitted to intensive care units. Medicines. 2016;95(31):1-5. Available from: https: //doi.org/10.1097/md.0000000000004376.

19. Jain S, Mittal A, Sharma SK, Upadhyay AD, Pandey RM, Sinha S. Predictors of Dengue-Related Mortality and Disease Severity in a Tertiary Care Center in North India. Open Forum Infectious Diseases; 2017. Available from: doi:10.1093/ofid/ofx056.
20. Routrayss, Mohanty DP, Mishrad, Clinicalprofile. IntensivecareUnit course and outcome of patients admitted in intensive care unit with dengue. Indian J Clin Anaesth . 2016;3(1):33-35.

21. Pinto RC, Dbd C, Bcd A, Sampaiovds, Passosrad C, Cfd. Mortality Predictors in Patients with Severe Dengue in the State of Amazonas, Brazil. PLoS One. 2016;11(8). Available from: https://doi.org/10.1371/journal.pone.0161884.

22. Ong A, Sandar M, Chen MI, Sin LY. Fatal dengue hemorrhagic fever in adults during a dengue epidemic in Singapore. Int $\mathbf{J}$ Infect Dis. 2007;11(3):263-267. Available from: https://dx.doi. org/10.1016/j.ijid.2006.02.012.

23. Pang J, Thein TL, Leo YS, Lye DC. Early clinical and laboratory risk factors of intensive care unit requirement during 2004-2008 dengue epidemics in Singapore: a matched casecontrol study. BMC Infect Dis. 2014;14(1):649-649. Available from: https://dx.doi.org/10.1186/s12879-014-0649-2.

24. Pang J, Salim A, Lee VJ, Hibberd ML, Chia KS, Leo YS, et al.. Diabetes with hypertension as risk factors for adult dengue hemorrhagic fever inapredominantlydengueserotype2epidemic:acasecontrolstudy; 2012. Available from: https://doi.org/10.1371/journal.pntd.0001641.

Copyright: (C) the author(s), 2020. It is an open-access article distributed under the terms of the Creative Commons Attribution License (CC BY 4.0), which permits authors to retain ownership of the copyright for their content, and allow anyone to download, reuse, reprint, modify, distribute and/or copy the content as long as the original authors and source are cited.

How to cite this article: Kumari PL. Clinical Profile and Outcome of Dengue Fever in Tertiary Care Hospital at Kerala. Acad. J Med. 2020;3(2):1-5.

DOI: dx.doi.org/10.47008/ajm.2020.3.2.1

Source of Support: Nil, Conflict of Interest: None declared. 